\title{
A Theoretical Study of Hydrodynamic Derivatives on Ship Maneuvering in Restricted Water
}

\author{
by Hironori Yasukawa*, Member
}

\begin{abstract}
Summary
Applying the method of matched asymptotic expansions, a prediction method of hydrodynamic derivatives on ship maneuvering in a shallow channel was developed. The numerical results are presented for a Series 60 model, $C_{b}=0.6$, and the effects of ship motion frequency, channel bank and water depth on the hydrodynamic derivatives are theoretically examined. It was found that in a shallow channel the hydrodynamic derivatives change appreciably with motion frequency, and that this change is due to the effect of shed vortices generated by ship maneuvering motion.
\end{abstract}

\section{Introduction}

In restricted water such as channel or canal a ship inevitably travels close to the channel bank or other ships, so that the hazard of collision will increase. Therefore, safe operation and effective control of the ship in such water require a sound knowledge of the hydrodynamic forces on it. This paper deals with the problems of ship maneuvering in a shallow channel. A theoretical model is developed for investigation of the nature and magnitude of such forces.

A brief review of the past analytical work on hydrodynamic forces in restricted water will be given first. Inoue and Kijima predicted the hydrodynamic derivatives on a rectangular plate moving obliquely in water channel by using the wing theory for small aspect ratio ${ }^{11}$. Exploiting the slender body assumptions, Newman presented closed form results for a spheroid moving near a wall2). Beck examined the hydrodynamic forces on a ship moving off the centerline of a channel by using the method of matched asymptotic expansions ${ }^{5)}$ similar to that employed by Tuck and Newman ${ }^{6}$. As these studies deal with the steady problem, it is not applied to the prediction of the accelerating terms of hydrodynamic forces and moments, i. e. added mass and moment of inertia. Yeung presented a calculation method of the unsteady interaction forces between two slender ships moving in a shallow water ${ }^{7)}$. This method takes into account the memory effect due to the shed vortices varying in response to relative position of two ships, and the numerical results showed good agreement with experimental results ${ }^{10)}$. Furthermore, Yeung and

* Nagasaki Experimental Tank, Mitsubishi Heavy Industries, Ltd.
Tan examined the hydrodynamic interactions of ships with fixed obstacle ${ }^{8)}$. However, this method deals with the problem of only ahead motion. Therefore, it is not applied to the calculation of hydrodynamic forces when a ship moves with swaying or yawing motion such as the case for zigzag motion.

In this paper, a calculation method of hydrodynamic forces acting on ship with lateral motion in a shallow channel is presented by extending the method presented by Yeung, and a prediction method of hydrodynamic derivatives on ship maneuvering is presented. Further, the effects of ship motion frequency, channel bank and water depth on the hydrodynamic derivatives are theoretically examined.

\section{Hydrodyanmic Derivatives on Ship Maneuvering in Restricted Water}

\subsection{Problem Formulation}

Let us consider the problem of a ship moving with forward velocity $U$ with lateral motion along the centerline of a channel as shown in Fig. 1. It is assumed that the cross section of the channel is rectangular and that water depth $h$ is uniform.

Two coordinate systems, $o_{0} x_{0} y_{0} z_{0}$ fixed in the fluid and oxyz moving with the ship, defined by the following transformations are employed.

$$
\left.\begin{array}{l}
x=x_{0}-U t, \\
y=y_{0}-W_{c} / 2, \\
z=z_{0},
\end{array}\right\}
$$

where $W_{c}$ is the channel width and $t$ represents time. The $z_{0^{-}}$and $z$-axes direct upwards.

The lateral motion of the ship is described by its displacement $\eta(x, t)$ from the $x$-axis. It is assumed that $\eta$ is small relative to the ship length and that this displacement is a slowly varying func- 


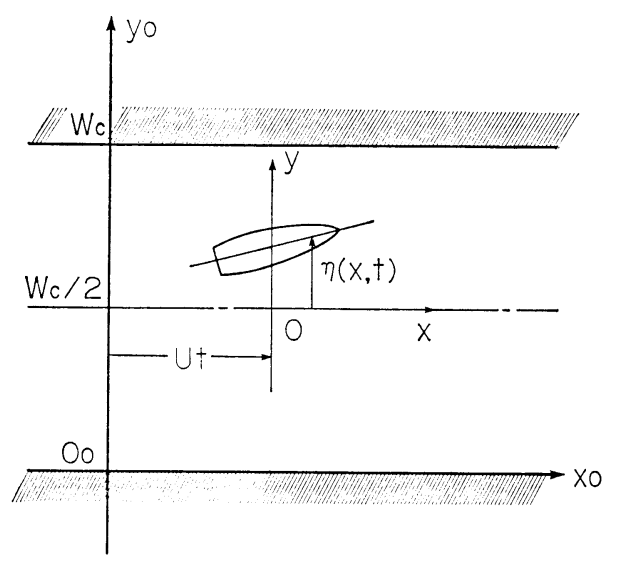

Fig. 1 Coordinate systems and notations

tion of $x$.

Let $\phi\left(x_{0}, y_{0}, z_{0} ; t\right)$ be the velocity potential describing the disturbances generated by the ship motion. With the assumption that the free surface can be treated as a rigid wall, the boundary-value problem for $\phi$ can be derived:

$$
\begin{gathered}
\left(\frac{\partial^{2}}{\partial x_{0}{ }^{2}}+\frac{\partial^{2}}{\partial y_{0}{ }^{2}}+\frac{\partial^{2}}{\partial z_{0}{ }^{2}}\right) \phi=0, \\
\frac{\partial \phi}{\partial n}=U n_{x}+\left(\frac{\partial \eta}{\partial t}-U \frac{\partial \eta}{\partial x}+\frac{\partial \phi}{\partial x_{0}} \frac{\partial \eta}{\partial x}\right) n_{y} \\
\text { on hull surface, } \\
\frac{\partial \phi}{\partial z}=0 \quad \text { at } \quad z= \pm h, \\
\frac{\partial \phi}{\partial y}=0 \quad \text { at } \quad y= \pm \frac{W_{c}}{2},
\end{gathered}
$$

where $n_{x}$ represents the $x$-component of the unit normal $n$ interior to hull surface, and $n_{y}$ the $y$ component.

In order to simplify the problem, the following assumptions are made.

(1) The length of the $\operatorname{ship} L$ is order of unity, and the breadth $B$ and the draft $d$ are small relative to the length :

$$
L=0(1), \quad B=0(\varepsilon), d=0(\varepsilon),
$$

where $\varepsilon$ is slenderness parameter.

(2) The water depth $h$ is shallow:

$$
h=0(\varepsilon) \text {. }
$$

(3) The channel bank is a large distance away from the ship ${ }^{5)}$ :

$$
s_{p}=0(1)
$$

where $s_{p}$ represents the distance between the ship and the channel bank.

\section{2 Inner Problem}

In the inner region close to the ship it follows that

$$
\frac{\partial}{\partial x}=0(1), \frac{\partial}{\partial y}, \frac{\partial}{\partial z}=0\left(\frac{1}{\varepsilon}\right) .
$$

By applying these to Eqs. (2), (3) and (4), the lead- ing-order potential $\Phi(y, z ; t)$ in this inner region can be shown to satisfy the following conditions.

$$
\begin{aligned}
& \left(\frac{\partial^{2}}{\partial y^{2}}+\frac{\partial^{2}}{\partial z^{2}}\right) \Phi=0, \\
& \frac{\partial \Phi}{\partial N}=U n_{x}+\left(\frac{\partial \eta}{\partial t}-U \frac{\partial \eta}{\partial x}\right) n_{y} \quad \text { on } \quad \Sigma_{B}, \\
& \frac{\partial \Phi}{\partial z}=0 \quad \text { at } \quad z= \pm h,
\end{aligned}
$$

where $N$ represents the unit two-dimensional interior normal to the section contour $\Sigma_{B}$ in the $y-z$ plane.

From the boundary condition (7), it is assumed that $\Phi$ is represented by the following expression.

$$
\Phi=U \Phi_{1}+V \Phi_{2}+\Phi^{*}+f(x, t),
$$

where

$$
V=\frac{\partial \eta}{\partial t}-U \frac{\partial \eta}{\partial x}
$$

and $\Phi_{1}$ and $\Phi_{2}$ are the unbounded shallow water flow potentials due to the longitudinal motion and the lateral motion respectively. $\Phi^{*}$ is an additional potential due to the partial diversion of the lateral flow. This diversion comes from the flow around the body ends and the effect of channel bank. $f$ is an arbitrary constant in this two-dimensional problem formulations.

$\Phi_{1}, \Phi_{2}$ and $\Phi^{*}$ are required to satisfy the following conditions.

$$
\left.\begin{array}{l}
\nabla^{2} \Phi_{1}=0, \\
\frac{\partial \Phi_{1}}{\partial N}=n_{x} \quad \text { on } \quad \Sigma_{B}, \\
\frac{\partial \Phi_{1}}{\partial z}=0 \quad \text { at } \quad z= \pm h, \\
\nabla^{2} \Phi_{2}=0, \\
\frac{\partial \Phi_{2}}{\partial N}=n_{y} \quad \text { on } \quad \Sigma_{B}, \\
\frac{\partial \Phi_{2}}{\partial z}=0 \quad \text { at } \quad z= \pm h, \\
\nabla^{2} \Phi^{*}=0, \\
\frac{\partial \Phi^{*}}{\partial N}=0 \quad \text { on } \quad \Sigma_{B}, \\
\frac{\partial \Phi^{*}}{\partial z}=0 \quad \text { at } \quad z= \pm h .
\end{array}\right\}
$$

Now let us consider the outer limit of the inner solution. The limit for $\Phi_{1}$ is given by Tuck $^{4)}$ as

$$
\lim _{|y| \gg \varepsilon} \Phi_{1}=-\frac{1}{4 h}\left(\frac{d S}{d x}\right)|y|,
$$

where $S(x)$ is the double body sectional area of the ship.

With the following approximation in Eq. (12)

$$
n_{y} \simeq N_{y}
$$

where $N_{y}$ represents the $y$-component of $N$, the limit for $\Phi_{2}$ is given by Newman ${ }^{3)}$ as

$$
\lim _{|y| \gg \varepsilon} \Phi_{2}= \pm C(x)
$$

where the \pm corresponds respectively to $y \gtrless 0$. 
Here $C(x)$ is the blockage coefficient introduced by Sedov for two-dimensional cascade problem ${ }^{9)}$.

Because of the presence of water bottom a dominant stream at the outer limit for $\Phi^{*}$ can be regarded as a lateral flow which is uniform along $z$. Therefore, it is assumed that $\Phi^{*}$ is represented by the following from.

$$
\Phi^{*}=V^{*}\left(y+\Phi^{* *}\right),
$$

where $V^{*}$ is an additional cross flow velocity to $V$ at the ship section. $\Phi^{* *}$ is a potential required to satisfy the following conditions so that $\Phi^{*}$ satisfies the Eq. (13).

$$
\left.\begin{array}{l}
\nabla^{2} \Phi^{* *}=0, \\
\frac{\partial \Phi^{* *}}{\partial N}=N_{y} \quad \text { on } \quad \Sigma_{B}, \\
\frac{\partial \Phi^{* *}}{\partial z}=0 \quad \text { at } \quad z= \pm h .
\end{array}\right\}
$$

Then, the outer limit for $\Phi^{* *}$ is the same as that for $\Phi_{2}$ :

$$
\lim _{|y| \gg \varepsilon} \Phi^{* *}= \pm C(x)
$$

Therefore, the outer limit for $\Phi^{*}$ can be written as

$$
\lim _{|y| \gg \varepsilon} \Phi^{*}=V^{*}(y \pm C(x)) .
$$

From Eqs. (14), (16) and (20) the outer limit of the inner solution can be expressed as

$$
\lim _{h \gg \varepsilon} \Phi=-\frac{U}{4 h}\left(\frac{d S}{d x}\right)|y| \pm V C+V^{*}(y \pm C)+f
$$

\section{3 Outer Problem}

In the outer region far away from the ship it follows that

$$
x, y=0(1), z=0(\varepsilon) .
$$

Then, the problem in the outer region corresponds to the two-dimensional problem in the horizontal plane ${ }^{4)}$.

As $\eta$ is small relative to the ship length, the leading-order potential $\phi_{o}\left(x_{0}, y_{o} ; t\right)$ can be represented by distribution of source and vortex along the $x$-axis.

$$
\begin{aligned}
\phi_{0}\left(x_{0}, y_{0} ; t\right)= & \frac{1}{2 \pi} \int_{-L / 2}^{L / 2} \sigma(\xi, t) G^{(\sigma)}\left(x_{0}, y_{0} ; \xi, \eta_{0}\right) d \xi \\
& +\frac{1}{2 \pi} \int_{-\infty}^{L / 2} \gamma(\xi, t) G^{(r)}\left(x_{0}, y_{0} ; \xi_{0}, \eta_{0}\right) d \xi
\end{aligned}
$$

where $\sigma$ and $\gamma$ are the distributed source and vortex strengths respectively. $\left(\xi_{o}, \eta_{0}\right)$ is the source or vortex point, and $\left(x_{0}, y_{0}\right)$ is a field point. $G^{(\sigma)}$ and $G^{(r)}$ will be referred to as the "obstacle Green functions" ${ }^{8)}$, which are defined as

$$
\begin{aligned}
& G^{(\sigma)}\left(x_{0}, y_{0} ; \xi_{0}, \eta_{0}\right) \\
& =\log \sqrt{\left(x_{0}-\xi_{0}\right)^{2}+\left(\overline{y_{0}}-\eta_{0}\right)^{2}}+H^{(\sigma)}\left(x_{0}, y_{0} ; \xi_{0}, \eta_{0}\right) \text {, } \\
& G^{(r)}\left(x_{0}, y_{0} ; \xi_{0}, \eta_{0}\right) \\
& =\tan ^{-1}\left(\frac{y_{0}-\eta_{0}}{x_{0}-\xi_{0}}\right)+H^{(r)}\left(x_{0}, y_{0} ; \xi_{0}, \eta_{0}\right),
\end{aligned}
$$

where $H^{(\sigma)}$ and $H^{(r)}$ are harmonic functions in the physical domain of interest and are constructed so as to satisfy the boundary condition on the channel bank. The constructions of $G^{(\sigma)}$ and $G^{(r)}$ may be found in the author's previous paper ${ }^{10)}$.

Let us consider the inner limit $(y \rightarrow \pm 0)$ of the outer solution. Making a Taylor expansion of $\phi_{o}$ for small values of $y$ and omitting the higher order terms, the following equation can be obtained.

$$
\begin{aligned}
& \lim _{y \rightarrow \pm 0} \phi_{0}(x, y ; t)=\frac{1}{2 \pi} \int_{-L / 2}^{L / 2} \sigma(\xi, t) \\
& \quad \times\left[\log |x-\xi|+H^{(\sigma)}(x, 0 ; \xi, 0)\right] d \xi \\
& \quad+\frac{1}{2 \pi} \int_{-\infty}^{L / 2} \gamma(\xi, t) H^{(\gamma)}(x, 0 ; \xi, 0) d \xi \\
& \quad \pm \frac{1}{2} \int_{x}^{L / 2} \gamma(\xi, t) d \xi+\frac{\sigma(x, t)}{2}|y| \\
& \quad+\left\{\frac{1}{2 \pi} \int_{-L / 2}^{L / 2} \sigma(\xi, t) \frac{\partial H^{(\sigma)}}{\partial y}(x, 0 ; \xi, 0) d \xi\right. \\
& \left.\quad+\frac{1}{2 \pi} \int_{-\infty}^{L / 2} \gamma(\xi, t)\left[\frac{1}{x-\xi}+\frac{\partial H^{(\gamma)}}{\partial y}(x, 0 ; \xi, 0)\right] d \xi\right\} y
\end{aligned}
$$

\section{4 Integral Equation}

The outer solution for the inner problem and the inner solution for the outer problem are required to match in the intermediate region. By matching terms of similar nature in Eqs. (21) and (25), the following equations can be obtained.

$$
\begin{aligned}
& f= \frac{1}{2 \pi} \int_{-L / 2}^{L / 2} \sigma(\xi, t) \log |x-\xi| d \xi \\
&+\frac{1}{2 \pi} \int_{-\infty}^{L / 2} \gamma(\xi, t) H^{(\gamma)} d \xi, \\
& \sigma=-\frac{U}{2 h}\left(\frac{d S}{d x}\right), \\
&\left(V^{*}+V\right) C=\frac{1}{2} \int_{x}^{L / 2} \gamma(\xi, t) d \xi, \\
& V^{*}=\frac{1}{2 \pi} \int_{-L / 2}^{L / 2} \sigma(\xi, t) \frac{\partial H^{(\sigma)}}{\partial y} d \xi \\
& \quad+\frac{1}{2 \pi} \int_{-\infty}^{L / 2} \gamma(\xi, t)\left[\frac{1}{x-\xi}+\frac{\partial H^{(\gamma)}}{\partial y}\right] d \xi .
\end{aligned}
$$

Eliminating $V^{*}$ from Eqs. (28) and (29), and substituting Eq. (27), the integral equation for $\gamma$ can be obtained as

$$
\begin{aligned}
& \frac{1}{2 C(x)} \int_{x}^{L / 2} \gamma(\xi, t) d \xi-V(x, t) \\
& =-\frac{U}{4 \pi h} \int_{-L / 2}^{L / 2}\left(\frac{d S}{d x}\right) \frac{\partial H^{(\sigma)}}{\partial y} d \xi \\
& \quad+\frac{1}{2 \pi} \int_{-\infty}^{L / 2} r(\xi, t)\left[\frac{1}{x-\xi}+\frac{\partial H^{(\gamma)}}{\partial y}\right] d \xi .
\end{aligned}
$$

In this equation, the first term on the left-hand side represents the effect of bottom clearance and $V$ is the lateral velocity defined by Eq. (10). The first term on the right-hand side represents the effect of the channel bank due to the source distribution and the second term is the contribution due to the vortex distribution including the effect of channel bank.

The solution of Eq. (30) should be augmented by 
the additional conditions that the pressure is continuous across the wake vortex, and that Kelvin's theorem is satisfied:

$$
\begin{aligned}
& r(x, t)=\gamma(x) \quad \text { for } \quad-\infty<x<-L / 2, \\
& \int_{-\infty}^{L / 2} \gamma(\xi, t) d \xi=0 .
\end{aligned}
$$

\subsection{Hydrodynamic Derivatives on Ship Ma- neuvering}

By applying Bernoulli's theorem to the equivalent two-dimensional ship in outer region, the linearized pressure jump $\Delta p$ across the $x$-axis is given by

$$
\Delta p=-\rho\left(\frac{\partial}{\partial t}-U \frac{\partial}{\partial x}\right) \Delta \phi_{0}
$$

where $\rho$ is the density of water. $\Delta \phi_{0}$, the jump of $\phi_{0}$, is the third term of Eq. (25):

$$
\Delta \phi_{0}=\int_{x}^{L / 2} \gamma(\xi, t) d \xi \text {. }
$$

The lateral force $Y$ and yawing moment $N$ acting on ship can be obtained by integrating the pressure difference over the length of ship and multiplying by water depth $h$ :

$$
\begin{aligned}
Y= & \rho h\left[\frac{\partial}{\partial t} \int_{-L / 2}^{L / 2} d x \int_{x}^{L / 2} \gamma(\xi, t) d \xi\right. \\
& \left.+U \int_{-L / 2}^{L / 2} \gamma(x, t) d x\right], \\
N= & \rho h\left[\frac{\partial}{\partial t} \int_{-L / 2}^{L / 2} x d x \int_{x}^{L / 2} \gamma(\xi, t) d \xi\right. \\
& \left.+U \int_{-L / 2}^{L / 2} x \gamma(x, t) d x\right] .
\end{aligned}
$$

Eqs. (35) and (36) coincide with the equations presented by Yeung) excluding the effect of lateral motion, because the assumption of small lateral motion is employed in the present method.

On the other hand, in conventional maneuvering problem, $Y$ and $N$ used to be expressed as the sum of the terms in proportion to lateral acceleration $\dot{v}$, angular acceleration $\dot{r}$, lateral velocity $v$, and angular velocity $r$ :

$$
\begin{aligned}
& Y=Y_{\dot{v}} \dot{v}+Y_{\dot{r}} \dot{r}+Y_{v} v+Y_{r} r, \\
& N=N_{\dot{v}} \dot{v}+N_{\dot{r}} \dot{r}+N_{v} v+N_{r} r,
\end{aligned}
$$

where $Y_{\dot{v}}, Y_{\dot{r}}, Y_{v}, Y_{r}, N_{\dot{v}}, N_{\dot{r}}, N_{v}$ and $N_{r}$ are so-called hydrodynamic derivatives on ship maneuvering.

Then, from Eqs. (35) through (38) the following equations can be written.

$$
\begin{aligned}
Y_{v} \dot{v} & +Y_{\dot{r}} \dot{r}+Y_{v} v+Y_{r} r \\
= & \rho h\left[\frac{\partial}{\partial t} \int_{-L / 2}^{L / 2} d x \int_{x}^{L / 2} \gamma(\xi, t) d \xi\right. \\
& \left.+U \int_{-L / 2}^{L / 2} \gamma(x, t) d x\right], \\
N_{\dot{v}} \dot{v} & +N_{\dot{r}} \dot{r}+N_{v} v+N_{r} r \\
= & \rho h\left[\frac{\partial}{\partial t} \int_{-L / 2}^{L / 2} x d x \int_{x}^{L / 2} \gamma(\xi, t) d \xi\right. \\
& \left.+U \int_{-L / 2}^{L / 2} x \gamma(x, t) d x\right] .
\end{aligned}
$$

In order to express each term of the hydrodyna- mic derivatives, we consider the pure swaying and pure yawing motion after the manner of experimental technique ${ }^{11)}$.

(i) Pure Swaying Motion

In this motion, a ship is forced to sway without yawing motion. Therefore $v$ only changes periodically.

$$
v=v_{o} \cos \omega t, \quad r=0
$$

Then, the vortex strength $\gamma(x, t)$ must be a similar from as

$$
\gamma(x, t)=\gamma_{0}(x) \cos \left(\omega t-\varepsilon_{0}(x)\right),
$$

where $\omega$ is motion frequency, and $\varepsilon_{0}(x)$ phase shift.

Substituting Eqs. (41) and (42) into Eqs. (39) and (40), $Y_{i}, Y_{v}, N_{\dot{v}}$, and $N_{v}$ can be obtained as follows.

$$
\begin{aligned}
\left(\begin{array}{c}
\omega Y_{\dot{v}} \\
Y_{v}
\end{array}\right)= & \frac{\rho h}{v_{0}}\left[\omega \int_{-L / 2}^{L / 2} d x \int_{x}^{L / 2} \gamma_{0}(\xi)\left(\begin{array}{l}
\cos \varepsilon_{0}(\xi) \\
\sin \varepsilon_{0}(\xi)
\end{array}\right) d \xi\right. \\
& \left.\mp U \int_{-L / 2}^{L / 2} \gamma_{0}(x)\left(\begin{array}{l}
\sin \varepsilon_{0}(x) \\
\cos \varepsilon_{0}(x)
\end{array}\right) d x\right], \\
\left(\begin{array}{c}
\omega N_{\dot{v}} \\
N_{v}
\end{array}\right)= & \frac{\rho h}{v_{0}}\left[\omega \int_{-L / 2}^{L / 2} x d x \int_{x}^{L / 2} \gamma_{0}(\xi)\left(\begin{array}{c}
\cos \varepsilon_{0}(x) \\
\sin \varepsilon_{0}(x)
\end{array}\right) d \xi\right. \\
& \left.\mp U \int_{-L / 2}^{L / 2} x \gamma_{0}(x)\left(\begin{array}{l}
\sin \varepsilon_{0}(x) \\
\cos \varepsilon_{0}(x)
\end{array}\right) d x\right] .
\end{aligned}
$$

(ii) Pure Yawing Motion

In this motion the ship is forced to yaw and sway as its drift angle is always kept to be zero. Therefore $r$ only changes periodically.

$$
r=r_{o} \cos \omega t, v=0
$$

Then, $Y_{\dot{r}}, Y_{r}, N_{\dot{r}}$ and $N_{r}$ can be written as follows.

$$
\begin{aligned}
\left(\begin{array}{c}
\omega Y_{\dot{r}} \\
Y_{r}
\end{array}\right)= & \frac{\rho h}{r_{0}}\left[\omega \int_{-L / 2}^{L / 2} d x \int_{x}^{L / 2} r_{0}(\xi)\left(\begin{array}{c}
\cos \varepsilon_{0}(\xi) \\
\sin \varepsilon_{0}(\xi)
\end{array}\right) d \xi\right. \\
& \left.\mp U \int_{-L / 2}^{L / 2} r_{0}(x)\left(\begin{array}{l}
\sin \varepsilon_{0}(x) \\
\cos \varepsilon_{0}(x)
\end{array}\right) d x\right], \\
\left(\begin{array}{c}
\omega N_{\dot{r}} \\
N_{r}
\end{array}\right)= & \frac{\rho h}{r_{0}}\left[\omega \int_{-L / 2}^{L / 2} x d x \int_{x}^{L / 2} r_{0}(\xi)\left(\begin{array}{c}
\cos \varepsilon_{0}(\xi) \\
\sin \varepsilon_{0}(\xi)
\end{array}\right) d \xi\right. \\
& \left.\mp U \int_{-L / 2}^{L / 2} x \gamma_{0}(x)\left(\begin{array}{l}
\sin \varepsilon_{0}(x) \\
\cos \varepsilon_{0}(x)
\end{array}\right) d x\right] .
\end{aligned}
$$

If the $\gamma$ distribution can be obtained when a ship travels with pure swaying or yawing motion along the centerline of the channel, the hydrodynamic derivatives can be calculated from Eqs. (43), (44), (46) and (47).

\section{Results and Discussion}

The integral equation (30) with the auxiliary conditions (31) and (32) was solved using a vortex lattice method ${ }^{12)}$. The details of the numerical scheme are shown in Appendix. Basically the ship is divided into a number of segments, and all the vortex strength within the element is assumed to be concentrated at one point. The integral equation is then satisfied at control points so that a system of simultaneous equations results at each time step. Solving the simultaneous equations by matrix inversion yields the value of the vortex 


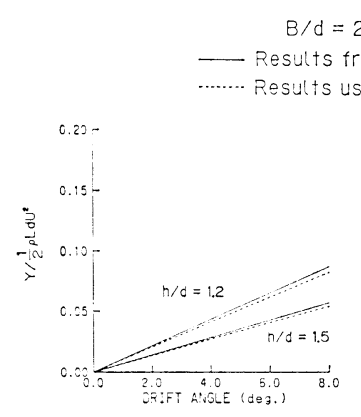

$B / d=2.0, F_{n}=0.0748$

- Results from Cohen and Beck ${ }^{15)}$

using present method

Fig. 2 Comparison of lateral forces and yawing moments for a mathematical ship in unbounded shallow water

Table 1 Comparison of hydrodynamic derivatives for a mathematical ship in unbounded shallow water Condition : $\mathrm{B} / \mathrm{d}=2.0, \mathrm{~F} n=0.0748$

\begin{tabular}{|l|c|c|c|c|}
\cline { 2 - 5 } \multicolumn{1}{c|}{} & \multicolumn{2}{|c|}{$\mathrm{h} / \mathrm{d}=1.2$} & \multicolumn{2}{c|}{$\mathrm{h} / \mathrm{d}=1.5$} \\
\hline & $-\mathrm{Y}_{\mathrm{V}}{ }^{\prime}$ & $-\mathrm{N}_{\mathrm{V}}{ }^{\prime}$ & $-\mathrm{Y}_{\mathrm{V}}{ }^{\prime}$ & $-\mathrm{N}_{\mathrm{V}}{ }^{\prime}$ \\
\hline Cohen and Beck $\left.{ }^{13}\right)$ & 0.623 & 0.600 & 0.410 & 0.417 \\
\hline Present Yethod & 0.591 & 0.588 & 0.389 & 0.427 \\
\hline
\end{tabular}

$$
\begin{aligned}
& Y_{v}{ }^{\prime}=Y_{v} / \frac{1}{2} \rho L d U^{2} \\
& N_{V}{ }^{\prime}=N_{V} / \frac{1}{2} \rho L^{2} d U^{2}
\end{aligned}
$$

strength.

Fig. 2 compares the lateral force and yawing moment obtained by using the present method with Cohen and Beck's calculations for a mathematical ship $^{15)}$. Cohen and Beck dealt with the steadystate problem based on a slender-body theory presented by $B \mathrm{Ck}^{5}$, and their calculated hydrodynamic forces showed qualitative agreement with experiments. The present results agree with Cohen and Beck's calculations using a quite different method. Table 1 shows the hydrodynamic derivatives, $Y_{v}^{\prime}$ and $N_{v}{ }^{\prime}$, obtained by analyzing these forces and moments. The agreement between both derivatives is also good. These checks validate the principles of the present algorithm as well as the computer program itself.

The numerical results are presented for a Series 60 model, $C_{b}=0.6$. Fig. 3 shows the sectional area curve of the ship. The blockage coefficient $C(x)$ was estimated by assuming that the section shape is rectangular and by using a formula given by Taylor ${ }^{13)}$. Fig. 4 shows $C(x)$ for various water depth ratios, $h / d$.

Figs. 5 through 8 show the hydrodynamic derivatives on ship maneuvering in unbounded shallow water. In these figures, the nondimensional derivatives are plotted for various $h / d(1.1,1.2,1.3$,

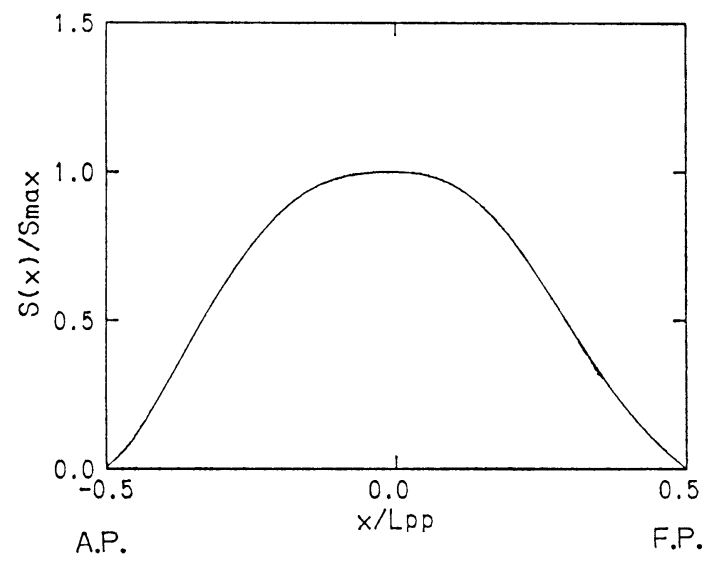

Fig. 3 Sectional area of a Series 60 model, $C_{b}=0.6$

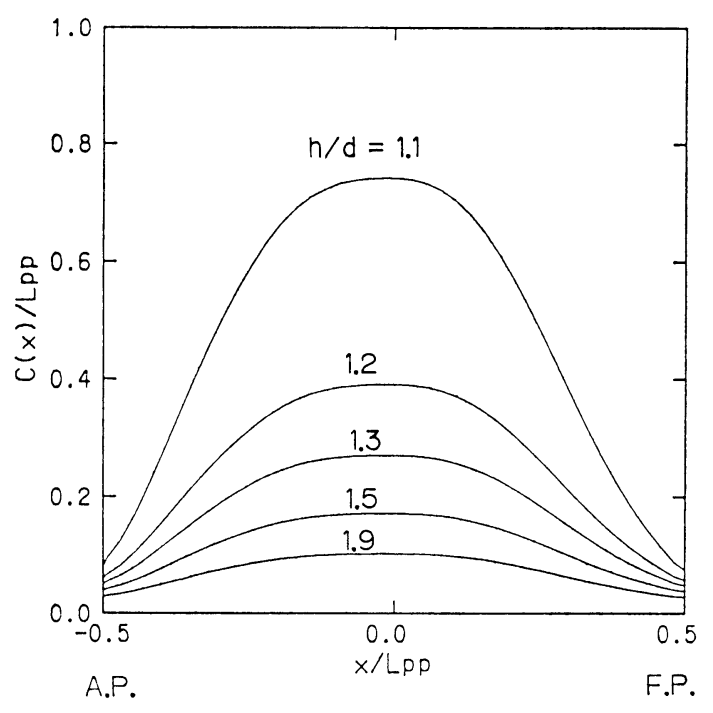

Fig. 4 Blockage coefficient curves for various $h / d$

1.5 and 1.9 ) versus nondimensional motion frequency $\omega^{\prime}$ defined as

$$
\omega^{\prime}=\omega \sqrt{\frac{L}{g}},
$$

where $g$ is the acceleration of gravity. The nondimensional derivatives are defined as follows.

$$
\left.\begin{array}{rl}
Y_{\dot{v}^{\prime}} & =Y_{\dot{v}} / \frac{1}{2} \rho L^{2} d U, \quad Y_{\dot{r}^{\prime}}=Y_{\dot{r}} / \frac{1}{2} \rho L^{3} d, \\
Y_{v^{\prime}} & =Y_{v} / \frac{1}{2} \rho L d U^{2}, \quad Y_{r^{\prime}}=Y_{r} / \frac{1}{2} \rho L^{2} d U, \\
N_{\dot{i}^{\prime}} & =N_{\dot{v}} / \frac{1}{2} \rho L^{3} d U, \quad N_{\dot{r}^{\prime}}=N_{\dot{r}} / \frac{1}{2} \rho L^{4} d, \\
N_{v}{ }^{\prime} & =N_{v} / \frac{1}{2} \rho L^{2} d U^{2}, \quad N_{r}^{\prime}=N_{r} / \frac{1}{2} \rho L^{3} d U .
\end{array}\right\}
$$

As can be seen from the figures, the derivatives undergo remarkable influence of water depth and these absolute values become larger in shallow 

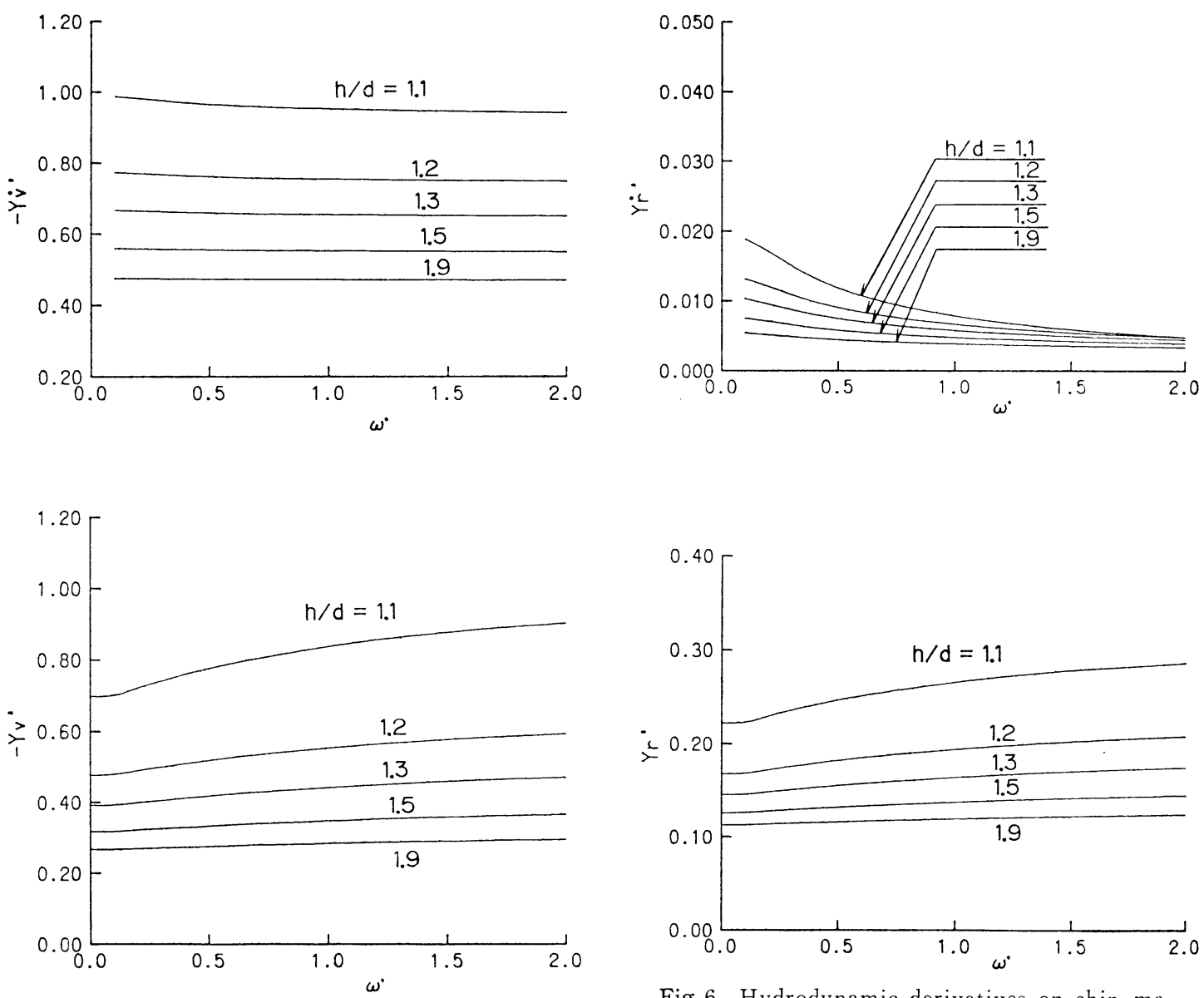

Fig. 5 Hydrodynamic derivatives on ship maneuvering, $Y_{v^{\prime}}^{\prime}$ and $Y_{v}^{\prime}$, for various $h / d$ in unbounded shallow water

water. In $h / d=1.9$ change of the derivatives for motion frequency can not be seen. However, in $h / d=1.1$ or 1.2 a remarkable change appears. When $\omega^{\prime}$ increases, $Y \dot{v}^{\prime}, Y_{r}^{\prime}, N_{v}^{\prime}$ and $N_{\dot{r}}^{\prime}$ become larger and $Y_{v}^{\prime}, Y_{\dot{r}^{\prime}}, N_{v^{\prime}}$ and $N_{r}^{\prime}$ become smaller. The behavior of the derivatives resembles the experimental results at low motion frequency obtained by Loeser ${ }^{14)}$.

Fig. 9 shows the strength of vortices shed behind the ship with pure swaying motion for various $h / d$ (1.1,1.3 and 1.9) versus ship position. When the water depth is shallow, the strength of shed vortices becomes larger. From these results, it can be considered that the vortices generated by unsteady maneuvering motion has influence on hydrodynamic forces in shallow water.

Figs. 10 through 13 compare the hydrodynamic derivatives obtained by using the present method with Loeser's experiments in unbounded shallow water. In these figures the derivatives are plotted versus $h / d$. The present results show qualitative

Fig. 6 Hydrodynamic derivatives on ship maneuvering, $Y_{\dot{r}^{\prime}}^{\prime}$ and $Y_{r}^{\prime}$, for various $h / d$ in unbounded shallow water

agreement with the experiments. However, some of the predictions are too low, particularly, $Y_{\dot{r}^{\prime}}$ and $Y_{r}^{\prime}$. This may be because of the higher order terms of velocity potential and viscous effects have been neglected.

Figs. 14 through 17 show the hydrodynamic derivatives in a shallow channel. In these figures, the derivatives are plotted for various $W_{c} / L(0.4$, $0.6,1.0$ and $\infty$ ) at a with $h / d=1.2$. As can be seen from the figures, absolute values of the derivatives become larger when channel width is small, and the effect of channel width on the derivatives is smaller than the effect of water depth. This tendency is the same as Fujino's experiments for Mariner ship model ${ }^{16)}$. However, the effect of channel width on the derivatives is small when compared with the trends observed in Fujino's experiments. Some improvements of the present method may be required for accurate prediction. The behavior of the derivatives for motion frequency is roughly similar to that in unbounded shallow 

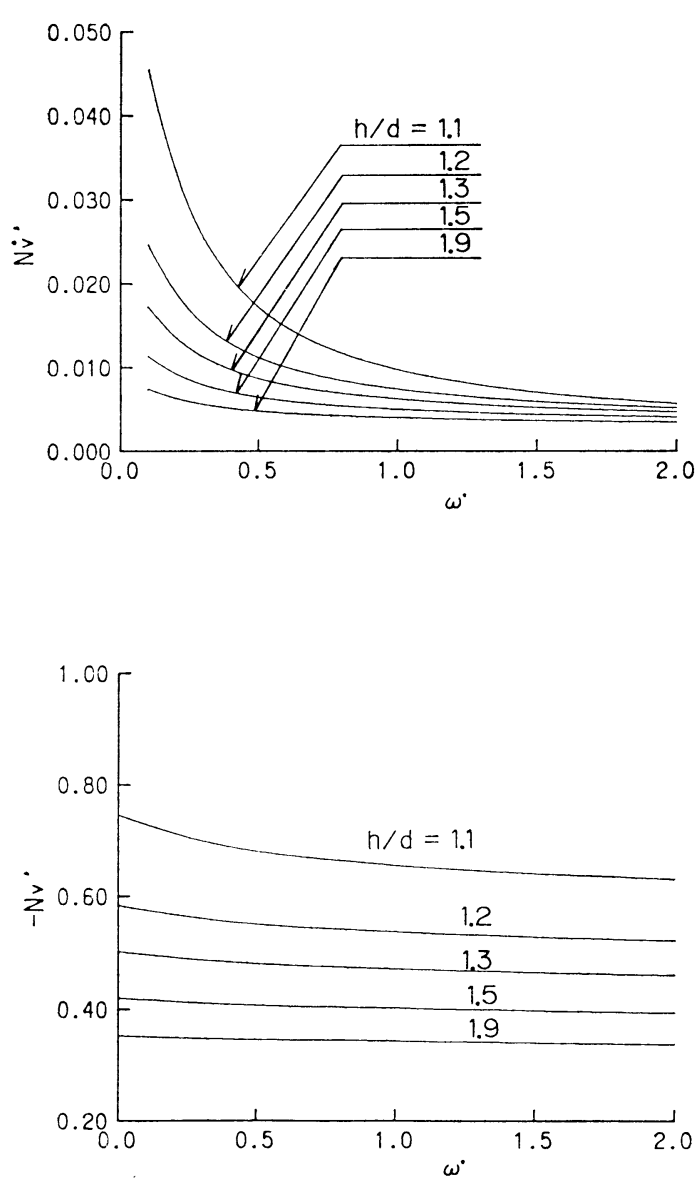

Fig. 7 Hydrodynamic derivatives on ship maneuvering, $N_{v}^{\prime}$ and $N_{v}^{\prime}$, for various $h / d$ in unbounded shallow water

water. However, there are the derivatives with different tendency at small frequency, and it is considered that it arises due to the complicated effect of mirror images of singular points because of the presence of channel walls.

In unbounded deep water, it has been considered that the change of the derivatives for motion frequency is small when ship moves slowly. However, in a shallow channel, it was found that the change for motion frequency appreciably appears due to the effect of shed vortices generated by ship maneuvering motion.

\section{Concluding Remarks}

A prediction method of hydrodynamic derivatives on ship maneuvering in a shallow channel was presented. The theoretical results showed that in a shallow channel the hydrodynamic derivatives change appreciably with motion frequency, and that this change is due to the effect of shed vortices generated by ship maneuvering motion.
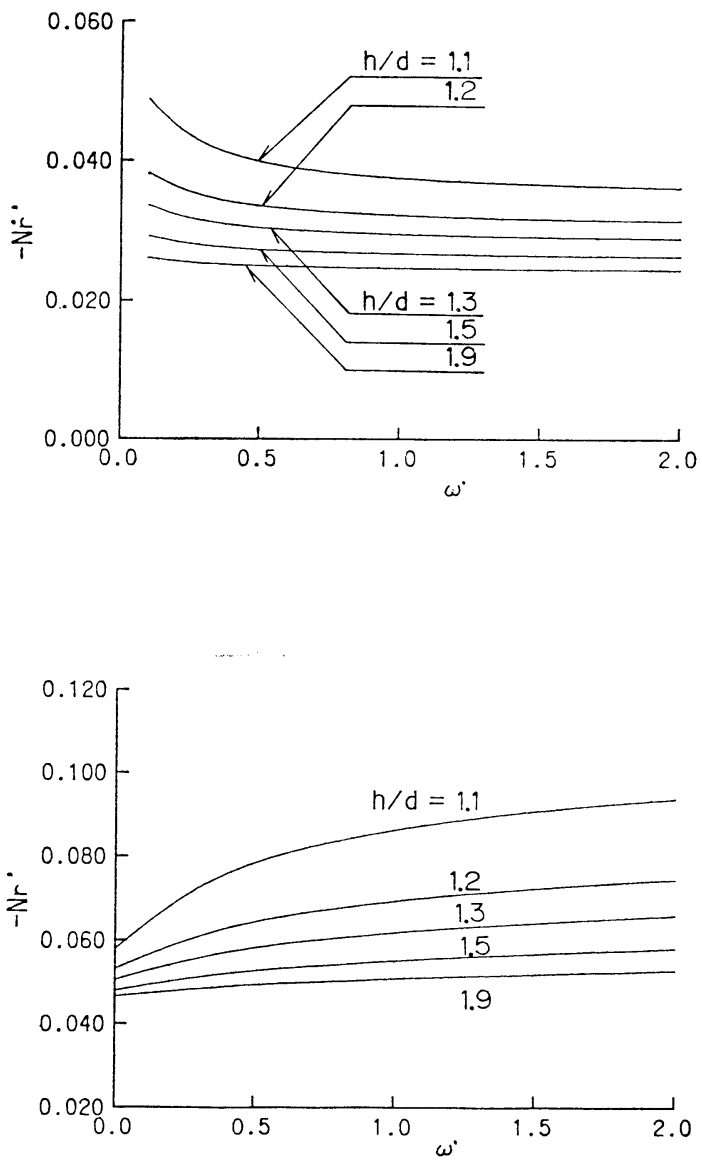

Fig. 8 Hydrodynamic derivatives on ship maneuvering, $N_{\dot{r}^{\prime}}$ and $N_{r}^{\prime}$, for various $h / d$ in unbounded shallow water

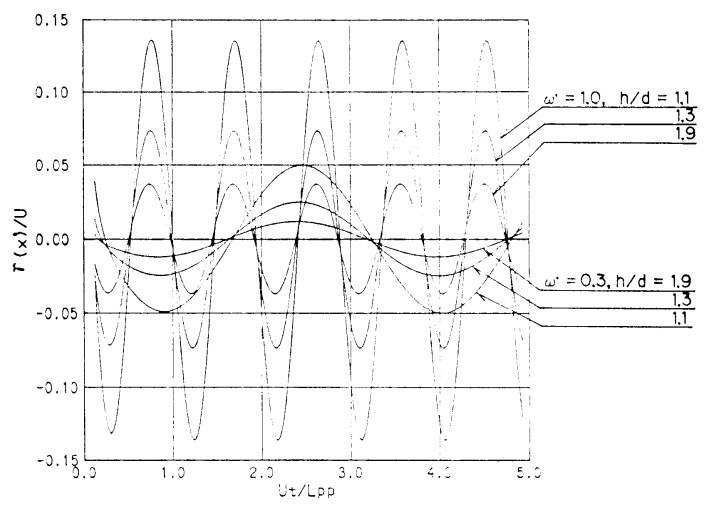

Fig. 9 Strength of the vortices shed behind a ship in unbounded shallow water

When we estimate the ship maneuverability by simulation method, the hydrodynamic derivatives are usually regarded as constant for the various ship motion. As described above, the effect of shed vortices generated by ship motion on the derivatives 


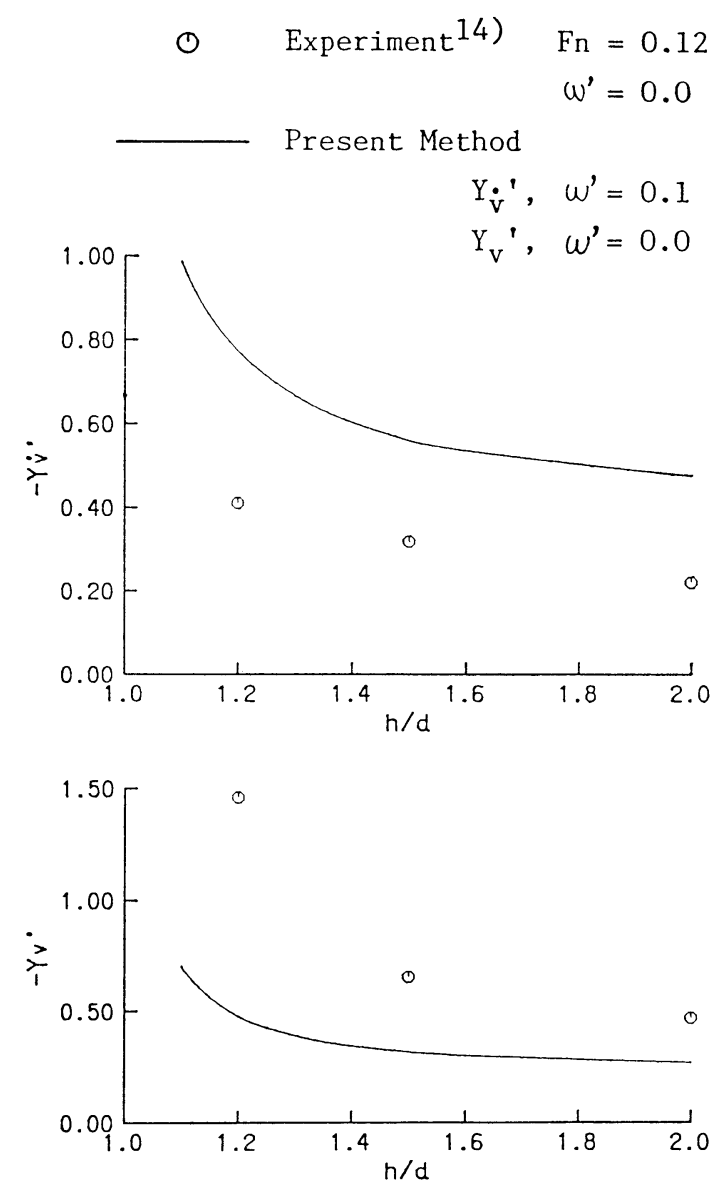

Fig. 10 Comparison of hydrodynamic derivatives on ship maneuvering, $Y_{v^{\prime}}^{\prime}$ and $Y_{v}^{\prime}$, in unbounded shallow water

is large in restricted water such as shallow channel, so that it is considered to be necessary to take into account the memory effect due to shed vortices on the derivatives for accurate simulation in restricted water.

Needless to say, further work is required for the improvement of the present method for accurate prediction and the verification of the method by experimental studies, especially for frequency dependency of hydrodynamic derivatives.

\section{Acknowledgment}

The author wishes to express his sincere gratitude to Professor K. Kijima of Kyushu University for his continuous encouragement and guidance. Thanks are also due to Dr.E. Baba, Senior Project Manager of Ship Hydrodynamics Laboratory, Nagasaki Research and Development center, MHI, and Dr. T. Nagamatsu, Project Manager of the same laboratory, for their valuable discussion. Thanks are also extended to all the members of the Nagasaki Experimental Tank for their cooperation.

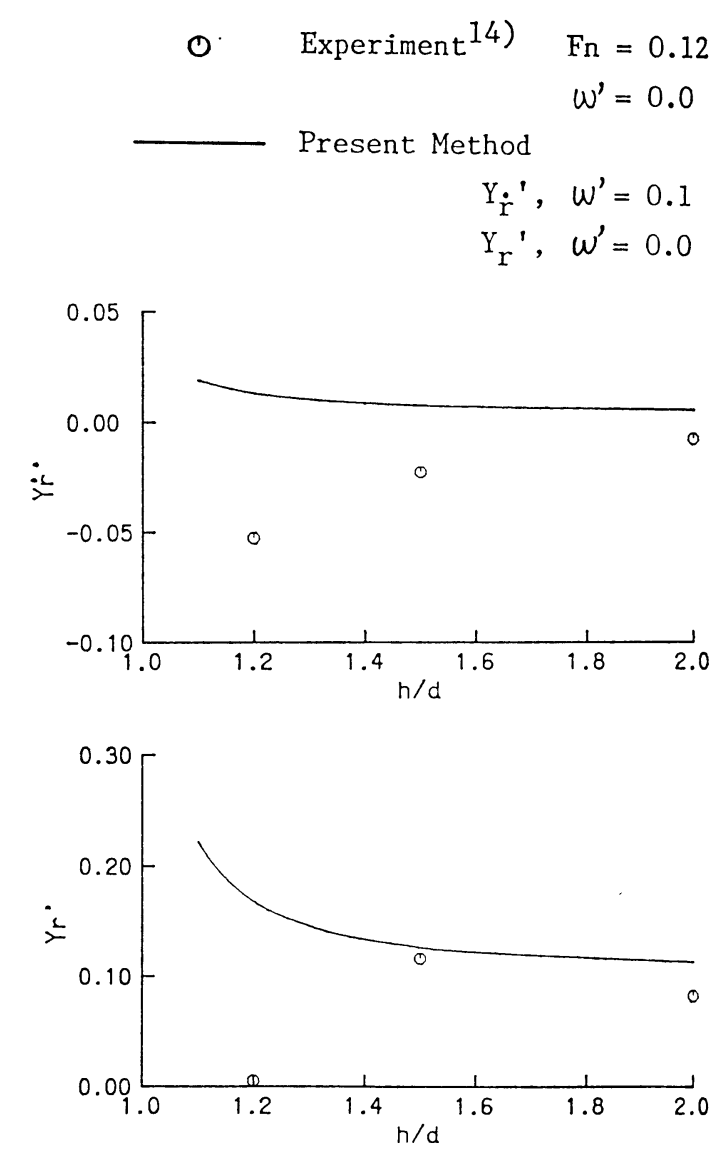

Fig. 11 Comparison of hydrodynamic derivatives on ship maneuvering, $Y_{r}{ }^{\prime}$ and $Y_{r}{ }^{\prime}$, in unbounded shallow water

\section{References}

1) Inoue, S. and Kijima, K.: Force Calculation of a Rectangular Plate Moving Obliquely in Water Channels, Transactions of the WestJapan Society of Naval Architects, No.39, 1969.

2) Newman, J. N.: The Force and Moment on a Slender Body of Revolution Moving Near a Wall, David Taylor Model Basin Rep. No. 2127, 1965.

3) Newman, J. N.: Lateral Motion of a Slender Body Between Two Parallel Wall, Journal of Fluid Mechanics, Vol.39, No. 1, 1969.

4) Tuck, E. O.: Shallow Water Flows Past Slender Bodies, Journal of Fluid Mechanics, Vol. 26, No. 1, 1966.

5) Beck, R. F.: Forces and Moments on a Ship Moving in a Shallow Channel, Journal of Ship Research, Vol.21, No.2, 1977.

6) Tuck, E. O. and Newman, J. N.: Hydrodynamic Interactions Between Ships, Proc. Tenth 


$$
\begin{aligned}
0 \quad \text { Experiment }^{14)} & \mathrm{Fn}_{n}=0.12 \\
\omega^{\prime} & =0.0
\end{aligned}
$$

Present Method
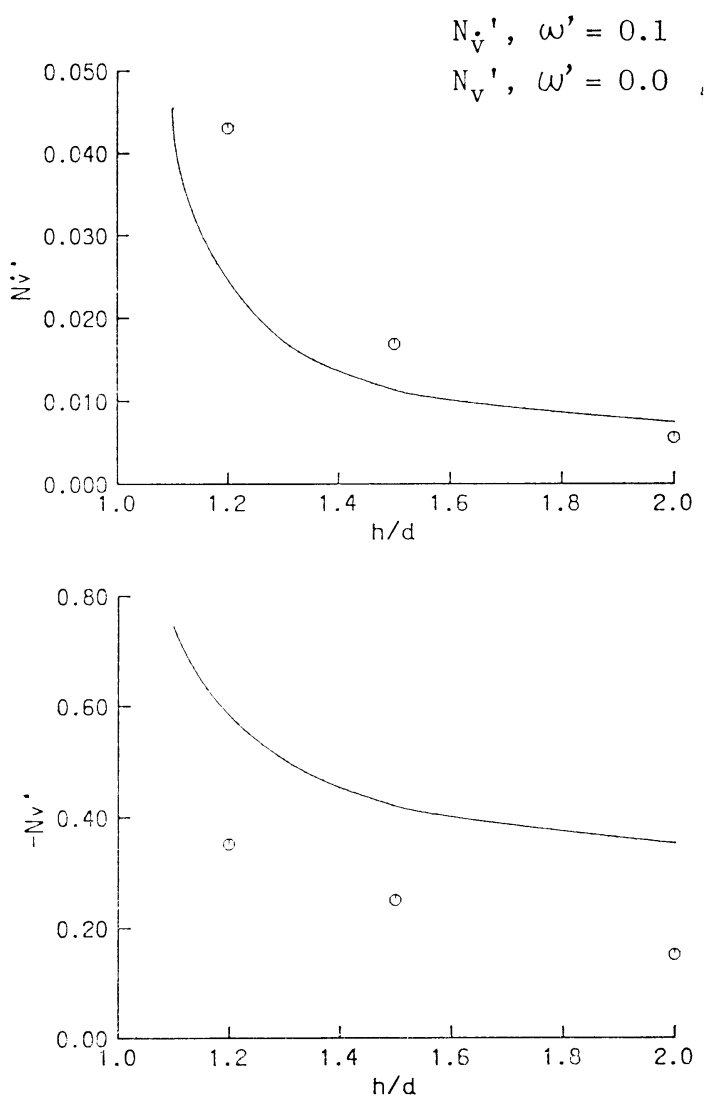

Fig. 12 Comparison of hydrodynamic derivatives on ship maneuvering, $N_{i^{\prime}}^{\prime}$ and $N_{v}^{\prime}$, in unbounded shallow water

Symposium on Naval Hydrodynamics, Cambridge, Mass., 1974 .

7) Yeung, R. W.: Interactions of Slender Ships in Shallow Water, Journal of Fluid Mechanics, Vol. 85, No.1, 1978.

8) Yeung, R. W. and Tan, W. T.: Hydrodynamic Interactions of Ships with Fixed Obstacles, Journal of Ship Research, Vol.24, No.1, 1980.

9) Sedov, L. I.: Two-Dimensional Problems in Hydrodynamics and Aerodynamics, Wily, New York, $196 \bar{J}$.

10) Kijima,K. and Yasukawa, H.: Mareuverability of Ships in Narrow Waterway, Journal cf the Society of Naval Architects of Japan, Tol. 156, 1984.

11) Motora, S. and Fujino, M.: On the Measurement of the Stability Derivatives by means of Forced Yawing Technique, Journal of the Society of Naval Architects of Japan, Vol.
(1) Experiment ${ }^{14)} \quad F_{n}=0.12$

$$
\omega^{\prime}=0.0
$$

\section{Present Method}
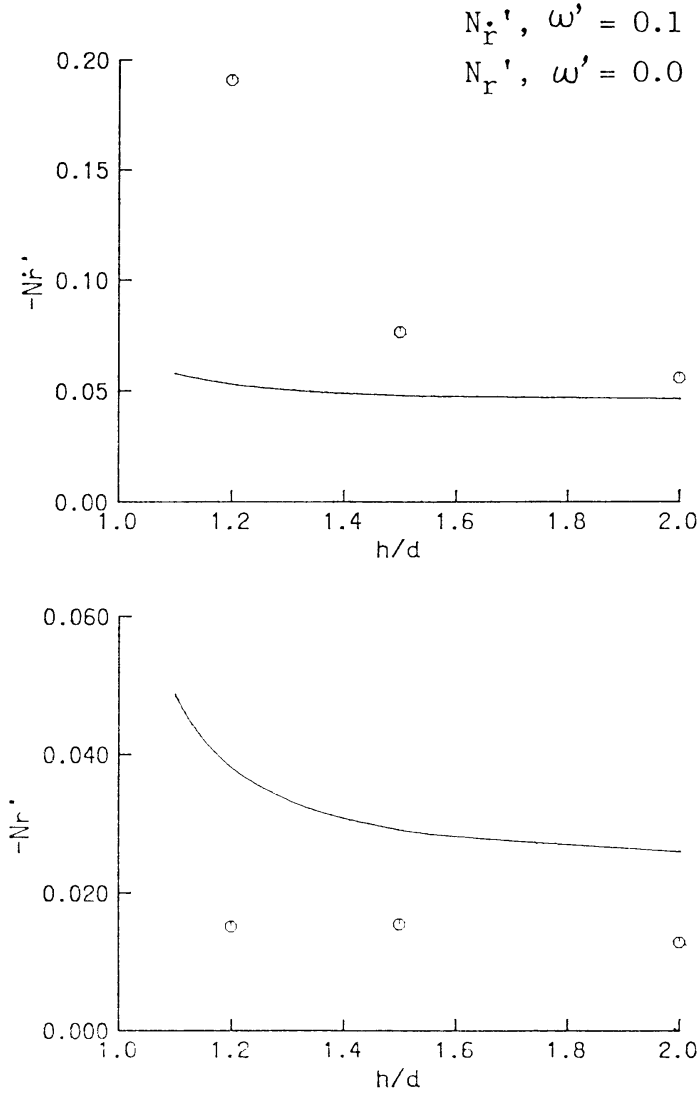

Fig. 13 Comparison of hydrodynamic derivatives on ship maneuvering, $N_{\dot{r}}^{\prime}$ and $N_{r}^{\prime}$, in unbounded shallow water

118, 1965.

12) James, R. M.: On the Remarkable Accuracy of the Vortex Lattice Method, Computer Methods in Applied Mechanics and Engineering, Vol. 1, 1972.

13) Taylor, P.J.: The Blockage Coefficient for Flow About an Arbitrary Body Immersed in a Channel, Journal of Ship Research, Vol. 17, No. 2, 1973.

14) Loeser, D. J.: Determination of Maneuvering Properties in Shallow Water by Impulse Response Techniques, Journal of Ship Research, Vol. 26, No. 1, 1982.

15) Cohen, S. B. and Beck, R.F.: Experimental and Theoretical Hydrodynamic Forces on a Mathematical Model in Confined Waters, Journal of Ship Research, Vol.27, No.2, 1983.

16) Fujino, M.: Studies on Maneuverability of Ships in Restricted Waters, Journal of the 

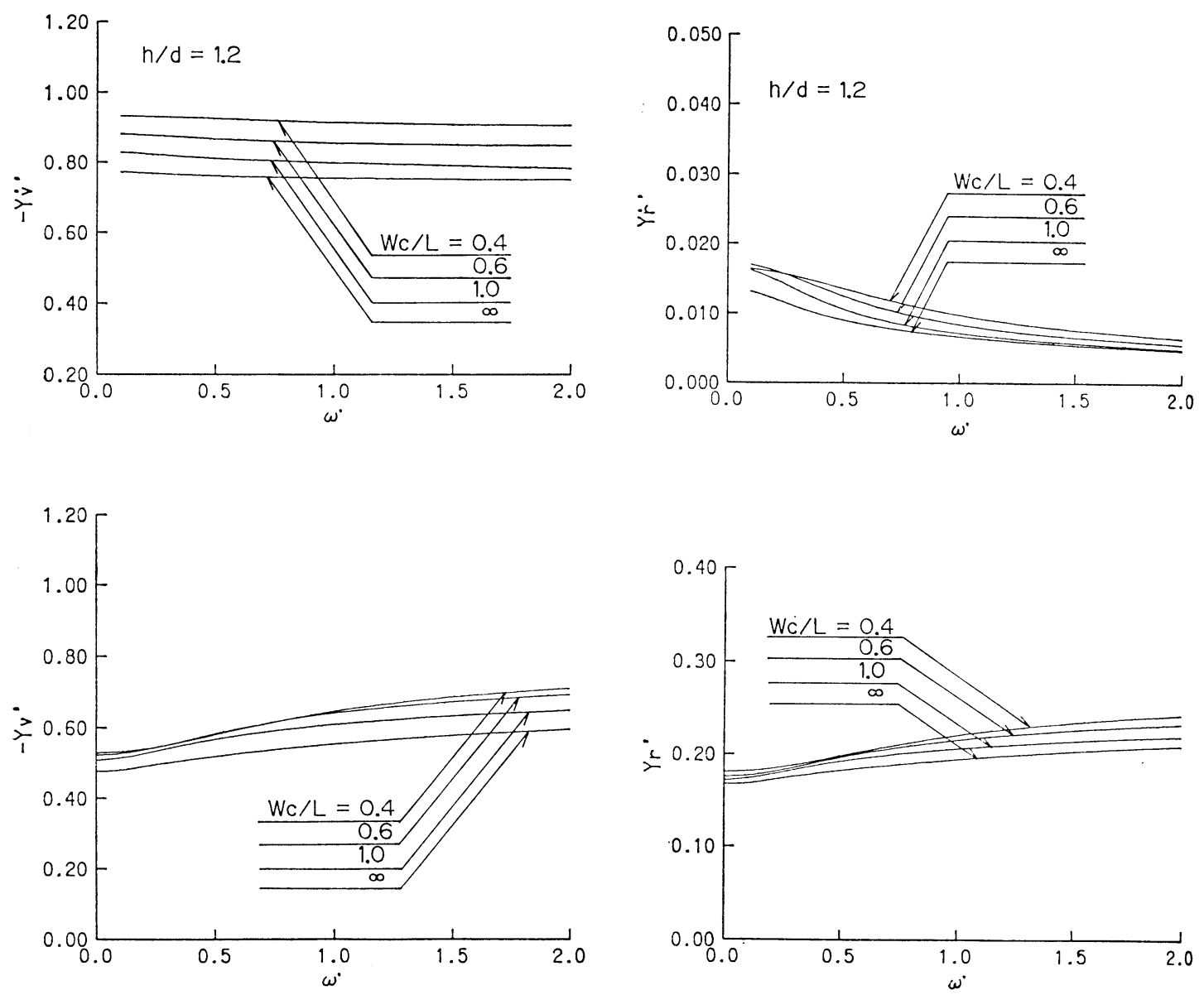

Fig. 14 Hydrodynamic derivatives on ship maneuvering, $Y_{v^{\prime}}^{\prime}$ and $Y_{v}^{\prime}$, for various $W_{c} / L$ in a shallow channel

Fig. 15 Hydrodynamic derivatives on ship maneuvering, $Y_{\dot{r}}^{\prime}$ and $Y_{r}^{\prime}$, for various $W_{c} / L$ in a shallow channel

Society of Naval Architects of Japan, Vol. 124, 1968.

\section{Appendix}

A ship is first divided into $M$ elements of equal length, $\Delta x$. Within each of these segments the vortex strength is assumed to take a certain form. Using these discrete-distribution assumption, the integral equation Eq. (30) can be transformed into a system of linear simultaneous equations. Let the vortex strength within the $i$ 'th element of the ship at time $t_{k}$ be denoted by $\gamma_{i}{ }^{(k)}, i=1,2, \cdots, M$. Let $\tilde{\gamma}_{i}, i=1.2, \cdots, k$ be the vortex strength in the wake element where $n=k$ corresponds to the wake element nearest the trailing edge of the ship.

Then Eq. (30) can be written as follows.

$$
\sum_{i=1}^{M} \mathrm{~A}_{i j} \gamma_{j}^{(k)}+\sum_{j=M+1}^{M+k} B_{i j} \tilde{\gamma}_{j}^{(k)}=D_{i}^{(k)}, \quad i=1,2, \cdots, M,
$$

where

$$
\begin{aligned}
B_{i j}= & -\frac{1}{\pi}\left[\frac{1}{x_{i}-\xi_{j}}+\frac{\partial H^{(r)}}{\partial y}\left(x_{i}, 0 ; \xi_{j}, 0\right)\right], \\
A_{i j}= & \begin{cases}B_{i j}+1 / C\left(x_{i}\right) & \text { for } \quad \xi_{j}>x_{i}, \\
B_{i j} & \text { for } \xi_{j}<x_{i},\end{cases} \\
D_{i}^{(k)}= & -\frac{U}{2 \pi h} \int_{-L / 2}^{L / 2}\left(\frac{d S}{d x}\right) \frac{\partial H^{(\sigma)}}{\partial y}\left(x_{i}, 0 ; \xi_{j}, 0\right) d \xi \\
& +2 V_{i}^{(k)},
\end{aligned}
$$

and $x_{i}$ is the control point located at the $i$ 'th segment, $\xi$, the vortex point on the $j$ 'th segment of the ship and its wake.

The additional equations can be obtained from Eqs. (31) and (32) in discretized form as follows.

$$
\begin{aligned}
& \tilde{\gamma}_{j}^{(k)}=\tilde{\gamma}_{j-1}{ }^{(k-1)} \quad \text { for } \quad \begin{array}{l}
k=1,2, \cdots \\
j=M+2, \cdots, M+k,
\end{array} \\
& \sum_{j=1}^{M} \gamma_{j}^{(k)}+\sum_{j=1}^{k} \tilde{\gamma}_{j}^{(k)}=0 .
\end{aligned}
$$

From the Eqs. (50), (54) and (55), the system of linear equations for the $M+1$ unknown can be 

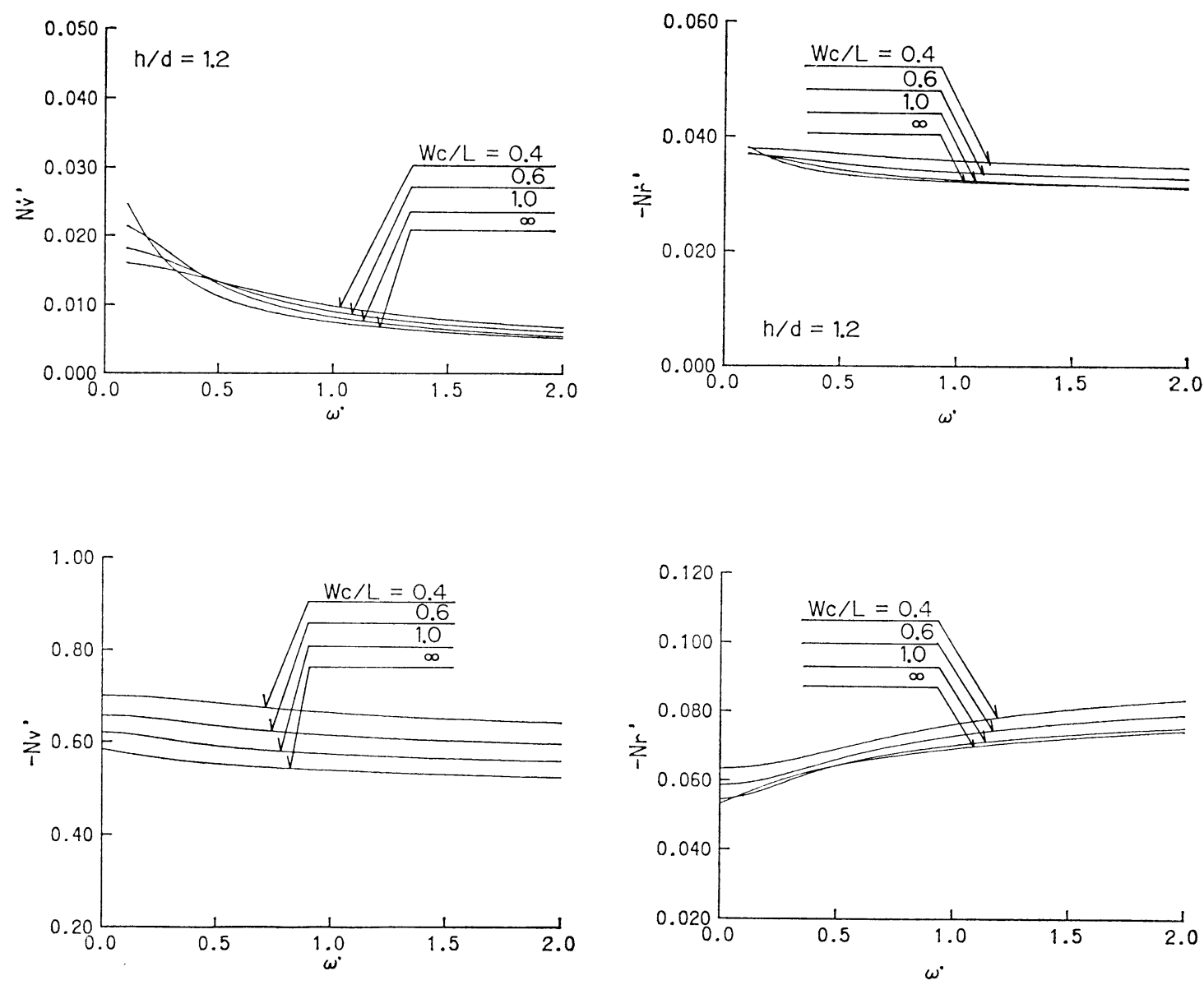

Fig. 16 Hydrodynamic derivatives on ship maneuvering, $N_{i}^{\prime}$ and $N_{v}^{\prime}$, for various $W_{c} / L$ in a shallow channel

Fig. 17 Hydrodynamic derivatives on ship maneuvering, $N_{\dot{r}}^{\prime}$ and $N_{r}^{\prime}$, for various $W_{c} / L$ in a shallow channel

obtained as

$\sum_{j=1}^{M} A_{i j} \gamma_{j}^{(i)}+B_{i, M+1} \tilde{\gamma}_{M+1}^{(k)}$

$=D_{i}^{(k)}-\sum_{j=\bar{M}+1}^{M+k-1} \tilde{\gamma}_{j}^{(k-1)} B_{i, j+1}, \quad i=1,2, \cdots, M$,

$\sum_{j=1}^{M} \gamma_{j}^{(k)}+\tilde{\gamma}_{M+1}^{(k)}=-\sum_{j=M+1}^{M+k-1} \tilde{\gamma}_{j}^{(k-1)}$.

In the present calculations, the vortex points and

control points were chosen to be $1 / 4$ and $3 / 4$ of the segment length respectively ${ }^{12)}$. The time-step $\Delta t$ was set as

$$
\Delta t=\Delta x / U,
$$

and the segment number of the ship $M$ was chosen to be 40 . The hydrodynamic derivatives were calculated by analyzing the $\gamma$ distribution from the 80 th time-step to the 300 th time-step excluding the effect of starting vortex. 